\title{
Effects of Chronic Mild Stress in Female Bax Inhibitor-1-Gene Knockout Mice
}

\author{
Zhi-Yan Sui ${ }^{1,2}$, Han-Jung Chae ${ }^{3}$, Guang-Biao Huang ${ }^{1,2}$, Tong Zhao ${ }^{1,2}$, Sushma Shrestha Muna ${ }^{1,2}$, Young-Chul \\ Chung,2
}

${ }^{1}$ Department of Psychiatry, Chonbuk National University Medical School \& Institute for Medical Sciences, ${ }^{2}$ Chonbuk National University Hospital \& Research Institute of Clinical Medicine, ${ }^{3}$ Department of Pharmacology, Chonbuk National University Medical School \& Institute for Medical Sciences, Jeonju, Korea

\begin{abstract}
Objective: The anti-apoptotic protein Bax inhibitor-1 $(\mathrm{Bl}-1)$ is a regulator of apoptosis linked to endoplasmic reticulum (ER) stress, and $\mathrm{Bl}-1^{-/-}$mice exhibit increased sensitivity to tissue damage. The purpose of this study was to investigate the role of $\mathrm{BI}-1$ in the pathogenesis of chronic mild stress (CMS)-induced depression-like behaviors in $\mathrm{BI}-1^{-/-}$mice.

Methods: We delivered CMS for 2 or 6 weeks in Bl-1-knockout and wild-type mice. Control groups of Bl-1-knockout and wild-type mice were left undisturbed. The measured parameters were sucrose consumption at weeks 1, 2, 3, 4, 5, and 6, spontaneous locomotion, and a forced swimming test (FST) at weeks 2 and 6.

Results: Significant decreases in sucrose consumption and increases in immobility time in the FST were observed in both stress groups compared with the non-stress groups. Interestingly, at week 2 , but not at week $6, \mathrm{Bl}-1^{-/-}-$stress mice showed less sucrose intake and greater immobility time than did $\mathrm{Bl}-1^{+/+}$-stress mice.

Conclusion: These results suggest that $\mathrm{Bl}-1$ may play role in protecting against the depressogenic effects of CMS in the short term, but not in the long term. Further study is required to deepen understanding of the role of $\mathrm{Bl}-1$ in protecting against depression.
\end{abstract}

KEY WORDS: Bax inhibitor-1; Chronic mild stress; Forced swimming test; Depression; Locomotor activity.

\section{INTRODUCTION}

Bax inhibitor-1 (BI-1) is an evolutionarily conserved, multi-transmembrane protein that resides in the endoplasmic reticulum (ER) and provides cytoprotective functions in both animals and plants. ${ }^{1,2)} \mathrm{BI}-1$ is expressed in normal cells and tissues including the heart, brain, placenta, liver, skeletal muscle, kidneys, and pancreas. ${ }^{3)}$ The cytoprotective function of BI-1 was originally discovered in cDNA library screens for human proteins capable of suppressing the death of yeast induced by ectopic expression of mammalian Bax protein. ${ }^{3)}$ BI-1 overexpression tagged to hemagglutinin or green fluorescent protein in several neuronal cell lines protects neuronal cells from oxygen-glucose deprivation or serum deprivation, a paradigm mimicking ischemia. ${ }^{4)}$ Cells isolated from $\mathrm{BI}-1^{-/-}$mice

\footnotetext{
Received: March 13, 2012 / Revised: May 10, 2012

Accepted: May 11, 2012

Address for correspondence: Young-Chul Chung, MD, PhD Department of Psychiatry, Chonbuk National University Medical School, 20, Geonji-ro, Deokjin-gu, Jeonju 561-712, Korea Tel: +82-63-250-2185, Fax: +82-63-275-3157 E-mail: chungyc@jbnu.ac.kr
}

exhibit hypersensitivity to apoptosis induced by ER stress. ${ }^{5)}$ Targeted ablation of the BI-1 gene in mice results in increased sensitivity to cell death induced by ischemia-reperfusion injury. ${ }^{6}$ In contrast, BI-1 is not essential for regulating programmed developmental cell death, as liver tissue of BI-1 deficient mice is histologically normal. ${ }^{7)}$ Although the exact mechanisms of the BI-1 anti-apoptotic function remain unclear, several lines of evidence suggest that it may be involved in the regulation of intra-ER $\mathrm{Ca}^{2+}{ }^{2}$ levels ${ }^{8)}$ and production of reactive oxygen species. ${ }^{9}$

Given the neuroprotective function of BI-1 for ER stress and oxygen-glucose deprivation, ${ }^{4)} \mathrm{BI}-1$ may be implicated in the pathogenesis of neurodegenerative disorders such as Alzheimer's disease, Parkinson's disease, and depression. However, up to now, no studies have investigated the role of BI-1 in these neurodegenerative and neuropsychiatric disorders. Chronic mild stress (CMS), an animal model of depression, has been reported to promote caspase-3 dependent neuronal apoptosis in the cerebral cortex ${ }^{10)}$ and increase serum corticosterone levels in female mice after 1 or 2 weeks of CMS. ${ }^{11)}$ We hypothesized that BI-1 may be involved in the diverse physio-

(c) This is an Open-Access article distributed under the terms of the Creative Commons Attribution Non-Commercial License (http://creativecommons.org/licenses/by-nc/3.0) which permits unrestricted non-commercial use, distribution, and reproduction in any medium, provided the original work is properly cited. 
logical or behavioral effects induced by CMS. The purpose of the present study was to investigate the role of BI-1 in the pathogenesis of CMS-induced depression-like behaviors using $\mathrm{BI}-1^{-/-}$mice.

\section{METHODS}

\section{Animals}

BI- ${ }^{-/-}(\mathrm{n}=18)$ and BI- $1^{+/+}$C57BL/6J female mice $(n=18,13$ weeks of age) were kindly provided by Dr. John C. Reed of the Stanford-Burnham Institute for Medical Research (La Jolla, CA, USA). Upon arrival, the mice were housed in groups of $3-5$ at $21 \pm 1^{\circ} \mathrm{C}$ and $55 \pm 5 \%$ humidity under a 12 -hour/12-hour light/dark cycle (lights on at 07:00). Food and water were available ad libitum. Mice were maintained in specific pathogen-free housing and cared for in accordance with the National Institute of Health guidelines for animal care. Permission for this study was given by the animal care and use committee of Chonbuk National University Graduate School of Medicine.

\section{Study Design}

After 10 days of initial acclimation to the laboratory, the mice were trained to drink a sucrose solution. Mice were exposed to two standard drinking bottles on alternating nights during week 1 ; one bottle contained $2.5 \%$ sucrose and the other held tap water. After this preliminary phase, mice were food and water deprived for 8 hours and then exposed to the sucrose solution and water from 18:00 until 09:00 to measure sucrose consumption. Three baseline sucrose consumption tests were performed, separated by 3 days. Each group of BI-1 $1^{+/+}$and BI-1 ${ }^{-/-}$mice was divided into two groups, control and stress groups, matched for sucrose consumption and body weight. This resulted in four different groups: $\mathrm{BI}-1^{+/+}$control and stress groups $(\mathrm{n}=4-6)$ and $\mathrm{BI}-1^{-1-}$ control and stress groups $(\mathrm{n}=4-6)$. The stress group was exposed to CMS, and the control group was left unchallenged. We initially conducted one experiment with a 6-week CMS treatment (experiment 1). Significant results were obtained after 2 weeks, so we conducted an additional short experiment with a 2-week CMS treatment (experiment 2). Behavioral tests, spontaneous locomotor activity, and a forced swimming test (FST), were performed between 08:00 and 14:00 after 2 and 6 weeks of exposure to CMS. No stressors were applied 15 hours before the tests (Fig. 1).

\section{CMS Procedure}

The CMS regimen used in this study was based on a protocol described previously, ${ }^{12,13)}$ with minor modifications. The CMS procedure was delivered to each mice and consisted of a variety of unpredictable mild environmental, social, and physical stressors, including confinement in a small tube (1 hour), an empty cage without sawdust (15 hours), water and food deprivation (15 hours), food restriction (about $50 \mathrm{mg}$ of food pellets, 3 hours), cage tilted at $45^{\circ}$ (1-3 hours), damp sawdust (about $200 \mathrm{ml}$ of water per $100 \mathrm{~g}$ of sawdust, 3 hours), paired housing in damp sawdust (18 hours), reversal of the light/dark cycle, 4-hour light/dark succession every 30 minutes (09:3011:30), and light (15-17 hours) dark (4-6 hours) (Table 1). To maximize the unpredictable nature of the stressors, two or three different stressors were applied in any 24 hours period in a pseudo-random and changing sequence throughout the experimental period. Control animals were single-housed during the time of CMS to match the condition but after that, group-housed in a different room and left undisturbed with the exception of general handling (e.g., regular cage cleaning and measuring body weight).

\section{Sucrose Consumption Test}

Sucrose intake and body weight were measured in the control and stress groups once per week on separate days during a 15-hour window (18:00-09:00) following 8 hours of food and water deprivation. The position of the two bottles was varied randomly from trial to trial. Stressors were not applied during the period of sucrose intake measurement, and the stressor of food and water deprivation was never conducted within the 15 hours before the sucrose consumption test or body weight measurements to avoid an effect of acute stress on the measures. Consumption
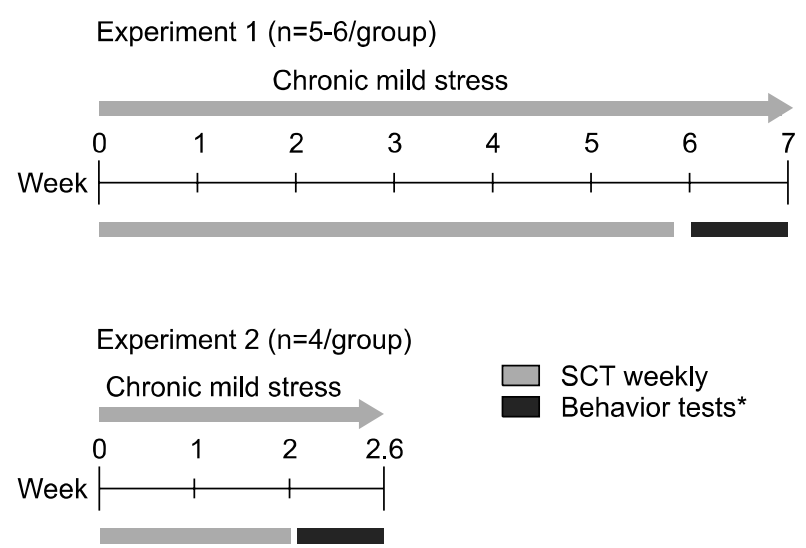

Fig. 1. Experiment design of the study. SCT, sucrose consumption test. *Behavior tests include locomotion and forced swimming test. 
Table 1. Procedure of chronic mild stress

\begin{tabular}{|c|c|c|c|c|c|c|c|c|c|}
\hline Week & Start & point & Monday & Tuesday & Wednesday & Thursday & Friday & Saturday & Sunday \\
\hline \multirow[t]{3}{*}{1} & $9: 00$ & a.m. & $\begin{array}{l}\text { Small tubes } \\
(1.5 \mathrm{~h})\end{array}$ & $\begin{array}{l}\text { Small tubes } \\
(1 \mathrm{~h})\end{array}$ & $\begin{array}{l}\text { Food restriction } \\
(3 \mathrm{~h})\end{array}$ & $\begin{array}{l}\text { Small tubes } \\
(1.5 \mathrm{~h})\end{array}$ & $\begin{array}{l}\text { Small tube } \\
(1 \mathrm{~h})\end{array}$ & $\begin{array}{l}\text { Reversal of the } \\
\text { light/dark cycle } \\
\text { (after 9:00 am) }\end{array}$ & $\begin{array}{l}\text { Reversal of the } \\
\text { light/dark cycle }\end{array}$ \\
\hline & $2: 00$ & p.m. & $\begin{array}{l}\text { Cages tilt at } 45^{\circ} \\
(1.5 \mathrm{~h})\end{array}$ & $\begin{array}{l}\text { Damp sawdust } \\
(3 \mathrm{~h})\end{array}$ & $\begin{array}{l}\text { Cages tilt at } 45^{\circ} \\
(1.5 \mathrm{~h})\end{array}$ & $\begin{array}{l}\text { Damp sawdust } \\
(3 \mathrm{~h})\end{array}$ & $\begin{array}{l}\text { Cages tilt at } 45^{\circ} \\
(1.5 \mathrm{~h})\end{array}$ & & \\
\hline & $6: 00$ & p.m. & $\begin{array}{l}\text { Empty cage } \\
(15 \mathrm{~h})\end{array}$ & $\begin{array}{l}\text { Water and food } \\
\text { deprivation } \\
(15 \mathrm{~h})\end{array}$ & $\begin{array}{l}\text { Empty cage } \\
(15 \mathrm{~h})\end{array}$ & $\begin{array}{l}\text { Cages tilt at } 45^{\circ} \\
(1 \mathrm{~h})\end{array}$ & $\begin{array}{l}\text { Water and food } \\
\text { deprivation } \\
(15 \mathrm{~h})\end{array}$ & $\begin{array}{l}\text { Reversal of the } \\
\text { light/dark cycle }\end{array}$ & $\begin{array}{l}\text { Reversal of the } \\
\text { light/dark cycle }\end{array}$ \\
\hline \multirow[t]{3}{*}{2} & $9: 00$ & a.m. & $\begin{array}{l}\text { End of reversal } \\
\text { cycle }(9: 00)\end{array}$ & $\begin{array}{l}\text { Cages tilt at } 45^{\circ} \\
(1.5 \mathrm{~h})\end{array}$ & $\begin{array}{l}\text { Small tube } \\
(1.5 \mathrm{~h})\end{array}$ & $\begin{array}{l}\text { Small tube } \\
(1.5 \mathrm{~h})\end{array}$ & $\begin{array}{l}\text { Cages tilt at } 45^{\circ} \\
(1.5 \mathrm{~h})\end{array}$ & $\begin{array}{l}4 \text { light/dark } \\
\text { succession } \\
\text { every } 30 \mathrm{~min} \\
(9: 30-11: 30)\end{array}$ & $\begin{array}{l}4 \text { light/dark } \\
\text { succession } \\
\text { every } 30 \mathrm{~min} \\
(9: 30-11: 30)\end{array}$ \\
\hline & $2: 00$ & p.m. & $\begin{array}{l}\text { Small tube } \\
(1.5 \mathrm{~h})\end{array}$ & $\begin{array}{l}\text { Small tube } \\
(1 \mathrm{~h})\end{array}$ & $\begin{array}{l}\text { Food restriction } \\
(3 \mathrm{~h})\end{array}$ & $\begin{array}{l}\text { Cages tilt at } 45^{\circ} \\
(1.5 \mathrm{~h})\end{array}$ & $\begin{array}{l}\text { Small tube } \\
(1 \mathrm{~h})\end{array}$ & & \\
\hline & $6: 00$ & p.m. & $\begin{array}{l}\text { Paired housing } \\
\text { in Damp } \\
\text { sawdust (18 h) }\end{array}$ & $\begin{array}{l}\text { Water and food } \\
\text { deprivation } \\
(15 \mathrm{~h})\end{array}$ & $\begin{array}{l}\text { Empty cage } \\
(15 \mathrm{~h})\end{array}$ & $\begin{array}{l}\text { Water and food } \\
\text { deprivation } \\
(15 \mathrm{~h})\end{array}$ & $\begin{array}{l}\text { Damp sawdust } \\
(3 \mathrm{~h})\end{array}$ & $\begin{array}{l}\text { Light }(15-17 \mathrm{~h}) \text {, } \\
\text { dark }(4-6 \mathrm{~h})\end{array}$ & $\begin{array}{l}\text { Light }(15-17 \mathrm{~h}) \\
\text { dark }(4-6 \mathrm{~h})\end{array}$ \\
\hline \multirow[t]{3}{*}{3} & 9:00 & a.m. & $\begin{array}{l}\text { Body weighing } \\
(10: 00)\end{array}$ & $\begin{array}{l}\text { Cages tilt at } 45^{\circ} \\
(3 \mathrm{~h})\end{array}$ & $\begin{array}{l}\text { Small tube } \\
(1 \mathrm{~h})\end{array}$ & $\begin{array}{l}\text { Food restriction } \\
(3 \mathrm{~h})\end{array}$ & $\begin{array}{l}\text { Damp sawdust } \\
(3 \mathrm{~h})\end{array}$ & $\begin{array}{l}\text { Reversal of the } \\
\text { light/dark cycle }\end{array}$ & $\begin{array}{l}\text { Reversal of the } \\
\text { light/dark cycle }\end{array}$ \\
\hline & $2: 00$ & p.m. & $\begin{array}{l}\text { Small tube } \\
(1.5 \mathrm{~h})\end{array}$ & $\begin{array}{l}\text { Damp sawdust } \\
(3 \mathrm{~h})\end{array}$ & $\begin{array}{l}\text { Cages tilt at } \\
45^{\circ}(3 \mathrm{~h})\end{array}$ & $\begin{array}{l}\text { Damp sawdust } \\
(3 \mathrm{~h})\end{array}$ & $\begin{array}{l}\text { Cages tilt at } 45^{\circ} \\
(1.5 \mathrm{~h})\end{array}$ & & \\
\hline & $6: 00$ & p.m. & $\begin{array}{l}\text { Food restriction } \\
(3 \mathrm{~h})\end{array}$ & $\begin{array}{l}\text { Empty cage } \\
(15 \mathrm{~h})\end{array}$ & $\begin{array}{l}\text { Small tube } \\
(1.5 \mathrm{~h})\end{array}$ & $\begin{array}{l}\text { Water and food } \\
\text { deprivation } \\
(15 \mathrm{~h})\end{array}$ & $\begin{array}{l}\text { Small tube } \\
(1.5 \mathrm{~h})\end{array}$ & $\begin{array}{l}\text { Reversal of the } \\
\text { light/dark cycle }\end{array}$ & $\begin{array}{l}\text { Reversal of the } \\
\text { light/dark cycle }\end{array}$ \\
\hline \multirow[t]{3}{*}{4} & $9: 00$ & a.m. & $\begin{array}{l}\text { Body weighing } \\
(10: 00)\end{array}$ & $\begin{array}{l}\text { Small tubes } \\
(1 \mathrm{~h})\end{array}$ & $\begin{array}{l}\text { Cages tilt at } 45^{\circ} \\
(1 \mathrm{~h})\end{array}$ & $\begin{array}{l}\text { Damp sawdust } \\
(3 \mathrm{~h})\end{array}$ & $\begin{array}{l}\text { Small tube } \\
(1.5 \mathrm{~h})\end{array}$ & $\begin{array}{l}\text { Reversal of the } \\
\text { light/dark cycle } \\
\text { (after 9:00 am) }\end{array}$ & $\begin{array}{l}\text { Reversal of the } \\
\text { light/dark cycle }\end{array}$ \\
\hline & $2: 00$ & p.m. & $\begin{array}{l}\text { Small tube } \\
(1 \mathrm{~h})\end{array}$ & $\begin{array}{l}\text { Cages tilt at } 45^{\circ} \\
(3 \mathrm{~h})\end{array}$ & $\begin{array}{l}\text { Food restriction } \\
(3 \mathrm{~h})\end{array}$ & $\begin{array}{l}\text { Small tube } \\
(1.5 \mathrm{~h})\end{array}$ & $\begin{array}{l}\text { Cages tilt at } 45^{\circ} \\
(3 \mathrm{~h})\end{array}$ & & \\
\hline & $6: 00$ & p.m. & $\begin{array}{l}\text { Empty cage } \\
(15 \mathrm{~h})\end{array}$ & $\begin{array}{l}\text { Water and food } \\
\text { deprivation } \\
(15 \mathrm{~h})\end{array}$ & $\begin{array}{l}\text { Cages tilt at } 45^{\circ} \\
(3 \mathrm{~h})\end{array}$ & $\begin{array}{l}\text { Cages tilt at } 45^{\circ} \\
(1.5 \mathrm{~h})\end{array}$ & $\begin{array}{l}\text { Damp sawdust } \\
(3 \mathrm{~h})\end{array}$ & $\begin{array}{l}\text { Reversal of the } \\
\text { light/dark cycle }\end{array}$ & $\begin{array}{l}\text { Reversal of the } \\
\text { light/dark cycle }\end{array}$ \\
\hline \multirow[t]{3}{*}{5} & $9: 00$ & a.m. & $\begin{array}{l}\text { End of reversal } \\
\text { cycle }(9: 00)\end{array}$ & $\begin{array}{l}\text { Cages tilt at } 45^{\circ} \\
(1.5 \mathrm{~h})\end{array}$ & $\begin{array}{l}\text { Food restriction } \\
(3 \mathrm{~h})\end{array}$ & $\begin{array}{l}\text { Small tube } \\
(1 \mathrm{~h})\end{array}$ & $\begin{array}{l}\text { Small tube } \\
(1.5 \mathrm{~h})\end{array}$ & $\begin{array}{l}4 \text { light/dark } \\
\text { succession } \\
\text { every } 30 \text { min } \\
(9: 30-11: 30)\end{array}$ & $\begin{array}{l}4 \text { light/dark } \\
\text { succession } \\
\text { every } 30 \text { min } \\
(9: 30-11: 30)\end{array}$ \\
\hline & $2: 00$ & p.m. & $\begin{array}{l}\text { Cages tilt at } 45^{\circ} \\
(3 \mathrm{~h})\end{array}$ & $\begin{array}{l}\text { Damp sawdust } \\
(3 \mathrm{~h})\end{array}$ & $\begin{array}{l}\text { Small tube } \\
(1 \mathrm{~h})\end{array}$ & $\begin{array}{l}\text { Damp sawdust } \\
(3 \mathrm{~h})\end{array}$ & $\begin{array}{l}\text { Cages tilt at } 45^{\circ} \\
(1.5 \mathrm{~h})\end{array}$ & & \\
\hline & $6: 00$ & p.m. & $\begin{array}{l}\text { Paired housing } \\
\text { in damp } \\
\text { sawdust (18 h) }\end{array}$ & $\begin{array}{l}\text { Water and food } \\
\text { deprivation } \\
(15 \mathrm{~h})\end{array}$ & $\begin{array}{l}\text { Paired housing } \\
\text { in damp } \\
\text { sawdust (18 h) }\end{array}$ & $\begin{array}{l}\text { Empty cage } \\
(15 \mathrm{~h})\end{array}$ & $\begin{array}{l}\text { Damp sawdust } \\
(3 \mathrm{~h})\end{array}$ & $\begin{array}{l}\text { Light }(15-17 \mathrm{~h}) \text {, } \\
\text { dark }(4-6 \mathrm{~h})\end{array}$ & $\begin{array}{l}\text { Light }(15-17 \mathrm{~h}) \\
\text { dark }(4-6 \mathrm{~h})\end{array}$ \\
\hline \multirow[t]{3}{*}{6} & $9: 00$ & a.m. & $\begin{array}{l}\text { Weighing } \\
(10 \mathrm{~h})\end{array}$ & $\begin{array}{l}\text { Cages tilt at } 45^{\circ} \\
(3 \mathrm{~h})\end{array}$ & $\begin{array}{l}\text { Cages tilt at } 45^{\circ} \\
(1.5 \mathrm{~h})\end{array}$ & $\begin{array}{l}\text { Small tube } \\
(1 \mathrm{~h})\end{array}$ & $\begin{array}{l}\text { Restriction } \\
(3 \mathrm{~h})\end{array}$ & $\begin{array}{l}\text { Reversal of the } \\
\text { light/dark cycle }\end{array}$ & $\begin{array}{l}\text { Reversal of the } \\
\text { light/dark cycle }\end{array}$ \\
\hline & $2: 00$ & p.m. & $\begin{array}{l}\text { Small tube } \\
(1 \mathrm{~h})\end{array}$ & $\begin{array}{l}\text { Food restriction } \\
(3 \mathrm{~h})\end{array}$ & $\begin{array}{l}\text { Small tube } \\
(1 \mathrm{~h})\end{array}$ & $\begin{array}{l}\text { Cages tilt at } 45^{\circ} \\
(3 \mathrm{~h})\end{array}$ & $\begin{array}{l}\text { Cages tilt at } 45^{\circ} \\
(1.5 \mathrm{~h})\end{array}$ & & \\
\hline & $6: 00$ & p.m. & $\begin{array}{l}\text { Water and food } \\
\text { deprivation } \\
(15 \mathrm{~h})\end{array}$ & $\begin{array}{l}\text { Paired housing } \\
\text { in damp } \\
\text { sawdust } \\
(18 \mathrm{~h})\end{array}$ & $\begin{array}{l}\text { Small tube } \\
(1 \mathrm{~h})\end{array}$ & $\begin{array}{l}\text { Damp sawdust } \\
(3 \mathrm{~h})\end{array}$ & $\begin{array}{l}\text { Empty cage } \\
(15 \mathrm{~h})\end{array}$ & $\begin{array}{l}\text { Reversal of the } \\
\text { light/dark cycle }\end{array}$ & $\begin{array}{l}\text { Reversal of the } \\
\text { light/dark cycle } \\
\text { End of reversal } \\
\text { cycle (after } 20 \mathrm{~h} \text { ) }\end{array}$ \\
\hline
\end{tabular}

h, hour(s); min, minute(s).

was measured by weighing the pre-weighed bottle at the end of the test. Intake was expressed in relation to the animal's body weight $(\mathrm{g} / \mathrm{g})$. Control mice were housed individually during the test.

\section{Spontaneous Locomotor Activity}

Locomotor activity was measured in an open field box
$(40 \times 50 \times 30 \mathrm{~cm})$ consisting of a white base and black walls, using a Smart video tracking system (Panlab S.L., Barcelona, Spain) in a softly illuminated $(40 \mathrm{~W})$, soundproof room. Each mouse was placed in the center of the open field apparatus, and the distance traveled $(\mathrm{cm})$, locomotor time (seconds), number of times crossing the center ( $50 \%$ of all area), and time spent in the center was re- 
corded at 10-minute intervals during a 30-minute period. The open field arena was thoroughly cleaned between tests.

\section{FST}

Each animal was placed in a beaker ( $18 \mathrm{~cm}$ diameter, 24 $\mathrm{cm}$ depth) filled with water (temperature $25-26^{\circ} \mathrm{C}$ ) to a height of $16 \mathrm{~cm}$. Immobility (floating with only small movements to keep balance) and swimming were scored throughout the last 4 minutes of the 6-minute test period. Scoring was done by a trained observer who was blind to the treatment paradigm.

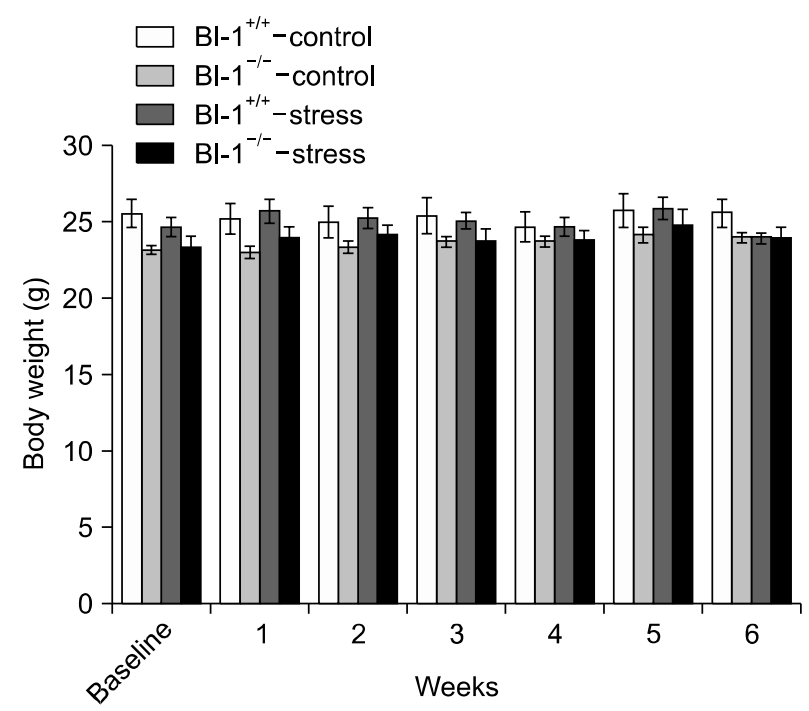

Fig. 2. Body weight of four experimental groups during 6-week treatment of chronic mild stress. BI-1, Bax inhibitor-1.

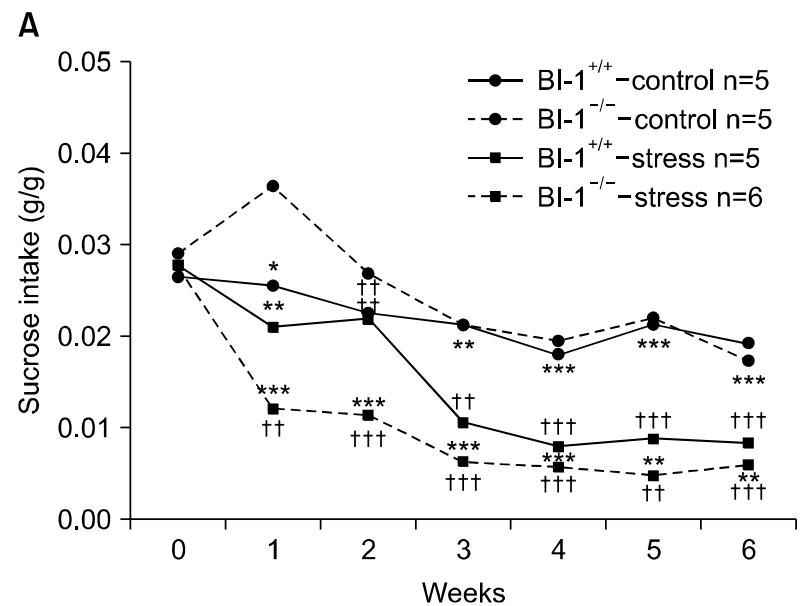

\section{Statistical Analysis}

All data are expressed as the mean \pm standard error of the mean. Body weight and sucrose consumption were analyzed with a repeated-measures two-way analysis of variance (ANOVA). Individual measures of body weight and sucrose consumption at each time point, locomotor activity, and FST immobility times were compared among the groups using a one-way ANOVA followed by Tukey's post hoc test. Differences of $p<0.05$ were considered significant.

\section{RESULTS}

No significant difference in the main effect of group $(F[3,17]=1.373, p=0.285)$, but significant differences in the main effect of time $(F[6,102]=6.480, p<0.001)$ and a group $\times$ time interaction $(F[18,102]=2.652, p=0.001)$ were observed for body weight (Fig. 2). Further analyses comparing the baseline and 6-week values among the groups revealed no significant differences.

\section{Sucrose Consumption}

The ANOVA yielded a significant main effect of group $(F[3,17]=92.562, p<0.001)$ and time $(F[6,102]=44.123$, $p<0.001)$ and a significant group $\times$ time interaction $(F[18,102]=4.460, p<0.001)$ for sucrose consumption (Fig. 3A, 3B). A post hoc group analysis demonstrated significant differences between the $\mathrm{BI}-1^{+/+}$-control group vs. the $\mathrm{BI}-1^{+/+}$-stress group and between the BI-1 ${ }^{-/-}$stress and $\mathrm{BI}-1^{+/+}$-stress groups vs. the $\mathrm{BI}-1^{-/-}$-control group as well as between the BI- $1^{-/-}$-stress and the BI-1 ${ }^{-/-}$stress groups vs. the $\mathrm{BI}-1^{-/-}$-control group. The BI- $1^{+/+}$-

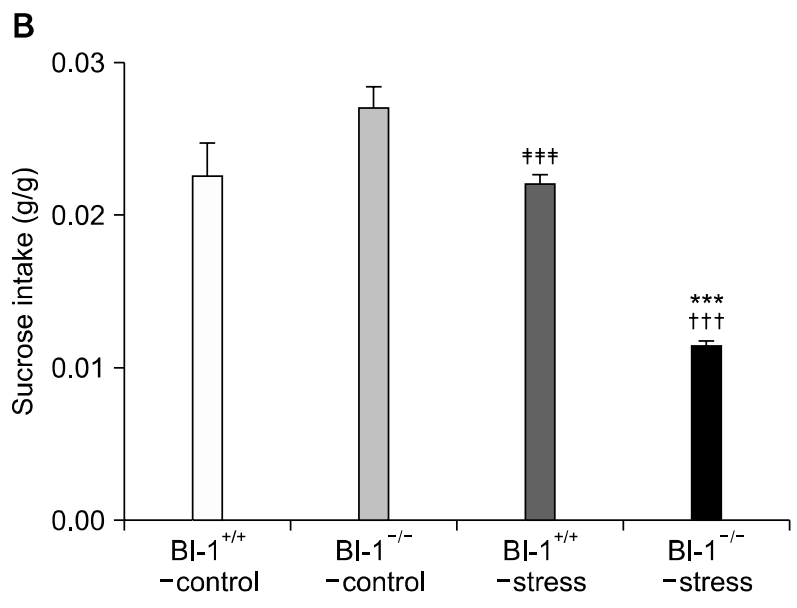

Fig. 3. (A) Effect of chronic mild stress on sucrose consumption over 6 weeks and (B) effect of chronic mild stress in sucrose consumption at week 2. ${ }^{*}$ Significant differences from Bl- ${ }^{-1-}$-control $\left({ }^{*} p<0.05,{ }^{* *} p<0.01,{ }^{* \star *} p<0.001\right) ;{ }^{\dagger}$ significant difference from $\mathrm{Bl}-1^{+/+}-\mathrm{control}\left({ }^{\dagger+} p<0.01\right.$, ${ }^{\dagger+\dagger} p<0.001$ ); ${ }^{\ddagger}$ significant difference from Bl-1 ${ }^{-1-}$-stress ( ${ }^{\ddagger \neq} p<0.01,{ }^{\ddagger \neq} p<0.001$ ). Bl-1, Bax inhibitor-1. 
Table 2. Spontaneous locomotor activities after 2- and 6-week treatment of chronic mild stress

\begin{tabular}{|c|c|c|c|c|}
\hline & $\begin{array}{l}\text { Distance traveled } \\
(\mathrm{cm})\end{array}$ & $\begin{array}{l}\text { Locomotor time } \\
\text { (seconds) }\end{array}$ & $\begin{array}{c}\text { Number of crossing } \\
\text { the center }\end{array}$ & $\begin{array}{l}\text { Time spent in the center } \\
\text { (seconds) }\end{array}$ \\
\hline \multicolumn{5}{|c|}{ 2-week CMS (n=4/group) } \\
\hline $\mathrm{Bl}-1^{+/+}$-control & $14,458.43 \pm 1,857.61$ & $1,172.70 \pm 142.63$ & $101.25 \pm 16.78$ & $343.1 \pm 76.39$ \\
\hline $\mathrm{Bl}-1^{-1-}$-control & $8,740.833 \pm 183.16$ & $655.96 \pm 137.03$ & $58.00 \pm 18.19$ & $345.23 \pm 182.53$ \\
\hline $\mathrm{Bl}-7^{+/+}$-stress & $17,004.15 \pm 3,825.87$ & $1,151.80 \pm 114.98$ & $99.25 \pm 31.06$ & $583.48 \pm 377.12$ \\
\hline $\mathrm{Bl}-1^{-1-}$-stress & $15,707.00 \pm 2,645.72$ & $1,175.35 \pm 126.07$ & $103.75 \pm 30.86$ & $289.78 \pm 125.13$ \\
\hline \multicolumn{5}{|c|}{ 6-week CMS ( $n=5-6 /$ group) } \\
\hline $\mathrm{Bl}-1^{+/+}$-control & $7,796.34 \pm 1,451.97$ & $793.76 \pm 78.67$ & $18.80 \pm 11.34$ & $80.52 \pm 49.06$ \\
\hline $\mathrm{Bl}-1^{-1-}-$ control & $10,212.21 \pm 1,084.61$ & $970.04 \pm 98.53$ & $57.40 \pm 5.56$ & $243.72 \pm 70.73$ \\
\hline $\mathrm{Bl}-1^{+/+}$-stress & $12,838.99 \pm 1,715.32$ & $1,066.32 \pm 86.01$ & $57.40 \pm 13.87$ & $277.86 \pm 39.92$ \\
\hline $\mathrm{Bl}-1^{-1-}$-stress & $14,966.96 \pm 1,289.70^{\dagger \dagger}$ & $1,144.33 \pm 67.77^{\dagger}$ & $57.00 \pm 16.93$ & $175.15 \pm 77.57$ \\
\hline
\end{tabular}

${ }^{+,{ }^{+}}$Significant differences from Bl- ${ }^{+/+}$-control $\left({ }^{\dagger} p<0.05,{ }^{\dagger+} p<0.01\right)$. Bl-1, Bax inhibitor-1.

A

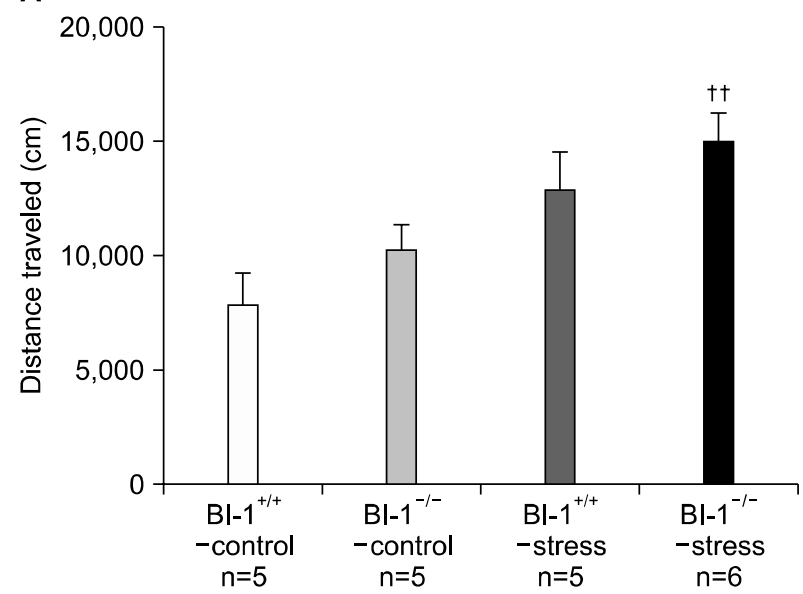

B

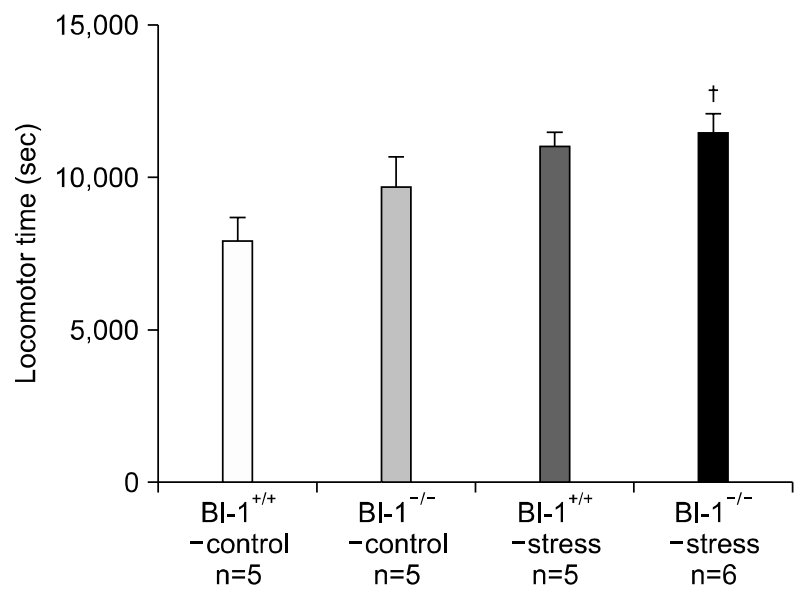

Fig. 4. Spontaneous locomotor activities ((A) distance traveled and (B) locomotor time) after 6 -week treatment of chronic mild stress. ${ }^{\dagger}$ Significant differences from $\mathrm{Bl}-1^{+/+}$-control $\left({ }^{\dagger} p<0.05,{ }^{+\dagger} p<0.01\right)$. Bl-1, Bax inhibitor-1.

stress and $\mathrm{BI}-1^{-/-}$-stress groups consumed significantly less sucrose than the BI- $1^{+/+}$-control and BI-1 ${ }^{-1-}$-control groups, starting from week 1 and throughout the experiment. No significant differences in sucrose consumption were observed between $\mathrm{BI}-1^{+/+}$-control BI-1 ${ }^{-/-}$control groups throughout the experiment, except at week 1 , when greater sucrose was consumed in the BI- $1^{-/-}$-control group than in the $\mathrm{BI}-1^{+/+}$-control group $(F=20.733$, $p=0.018$ ). Interestingly, at week 2 , less sucrose was consumed in the BI- $1^{-/-}$-stress group than in the BI- $1^{+/+}$. stress group $(F=28.592, p<0.001)$ (Fig. 3B).

\section{Spontaneous Locomotor Activity}

Significant differences were observed for distance traveled $(F=5.121, p=0.009)$ and locomotor time $(F=3.418$, $p=0.032$ ) among the groups in the 6-week CMS treatment, but not in the 2-week CMS treatment. Post hoc analyses revealed greater distances traveled and increased locomo- tor time in the $\mathrm{BI}-1^{-/-}$-stress group than in the $\mathrm{BI}-1^{+/+}$control group (Table 2, Fig. 4).

\section{FST}

Significant differences were observed in the immobility and swimming times among the groups for both the 2- and 6-week CMS treatments. Post hoc results showed a longer immobility time and a shorter swimming time in the $\mathrm{BI}-1^{+/+}$-stress and $\mathrm{BI}-1^{-/-}$-stress groups compared with the BI- $1^{+/+}$-control group and $\mathrm{BI}-1^{-/-}$-control group, respectively, in the 2-week and 6-week CMS treatments. In experiment 1, with a 2-week CMS treatment, significantly longer immobility time was observed in the BI-1 ${ }^{-/-}$-stress group than in the $\mathrm{BI}-1^{+/+}$-stress group $(F=42.465, p=0.003)$ (Fig. 5).

\section{DISCUSSION}

The role of BI-1 in producing depression-like behaviors 

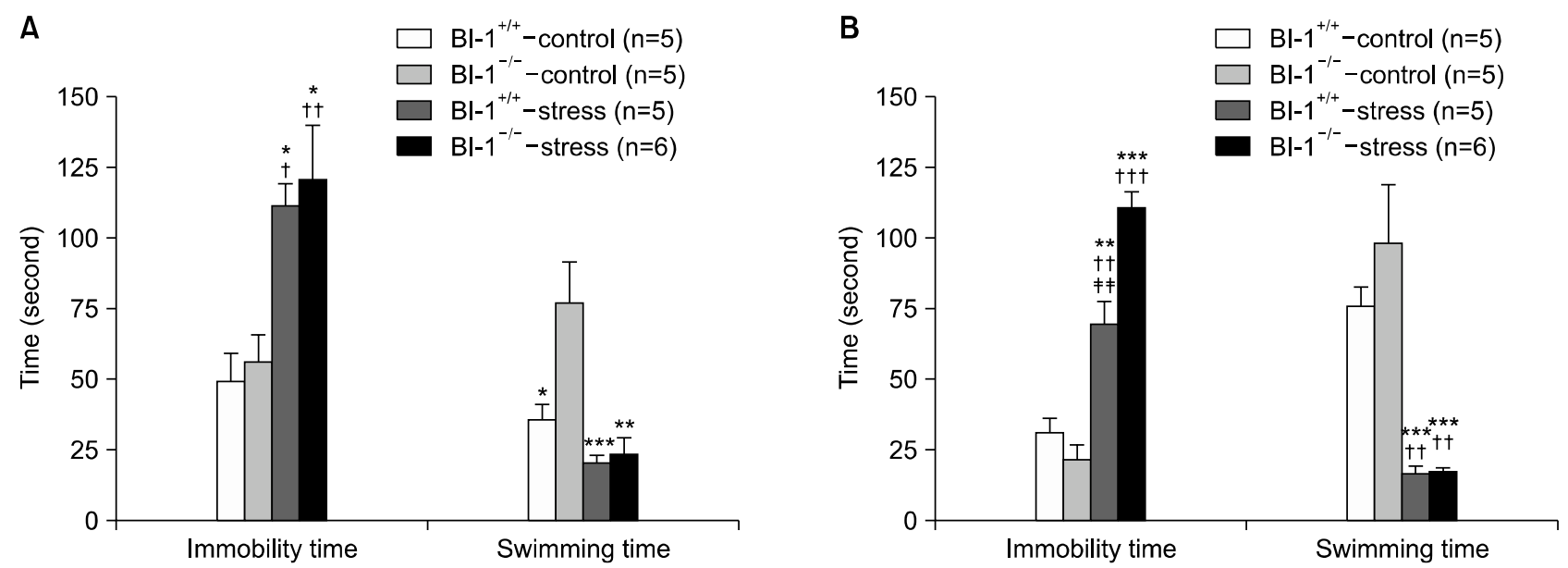

Fig. 5. Effects of chronic mild stress in the forced swimming test after 2-week (A) and 6-week (B) treatment of chronic mild stress. ${ }^{*}$ Significant differences from Bl- $1^{-1-}$-control $\left({ }^{*} p<0.05,{ }^{* *} p<0.01,{ }^{* * *} p<0.001\right)$; ${ }^{\dagger}$ significant difference from $\mathrm{Bl}-1^{+/+}-\mathrm{control}\left({ }^{\dagger} p<0.05,{ }^{\dagger+} p<0.01,{ }^{\dagger++} p\right.$ $<0.001) ;{ }^{\dagger}$ significant difference from Bl- $1^{-1-}$-stress $\left({ }^{\ddagger}{ }^{\dagger} p<0.01\right)$. BI-1, Bax inhibitor-1.

induced by CMS was investigated using BI-1-gene knockout mice. We showed that a BI-1 gene deficiency is implicated in producing more severe depression-like behaviors during a short-term CMS treatment (2-week), but not following long-term CMS treatment (6-week).

The CMS procedure decreased sucrose consumption significantly in both the wild-type and knockout mice, which was consistent with the majority of previous reports. ${ }^{14-16)}$ It should be noted, however, that different strains, such as dilute brown non-agouti (DBA/2) mice, do not show a decrease in sucrose consumption following $\mathrm{CMS}^{16)}$ Some researchers ${ }^{17,18)}$ have suggested that a reduction in sucrose intake may not be related directly to CMS per se, but secondary to stress-induced weight or appetite loss. However, as we did not observe significant weight changes during the CMS procedure, and because sucrose consumption was expressed as the absolute intake of sucrose per mouse body weight, that hypothesis seems invalid, at least for our study. Mixed findings, such as no change $^{14,16)}$ or an increase ${ }^{19)}$ in body weight, have been reported following a CMS procedure in $\mathrm{C} 57 \mathrm{BL} / 6 \mathrm{~J}$ mice. These discrepancies may be related to the severity of the CMS regimen and other experimental conditions employed in different studies. It is suggested that food and/or water deprivation should be removed from the CMS regimen or only water should be deprived before measuring sucrose consumption. The major finding of the present study was that the BI- $1^{-/-}$-stress group consumed a smaller amount of sucrose than was consumed by the $\mathrm{BI}-1^{+/+}$-stress group, suggesting that a BI-1 gene deficiency is involved in producing more severe anhedonic-like behavior following CMS. Thus, this result seems related to a neuroprotective function of BI-1. However, further studies are required to explore the molecular mechanisms of BI-1 regulation of the pathophysiological pathways induced by CMS. Interestingly, a transient increase in sucrose consumption in the $\mathrm{BI}-1^{-/-}$-control group compared with the $\mathrm{BI}-1^{+/+}$-control group was observed at week 1 . This finding, however, is difficult to interpret because both groups were under the same conditions. Given that food and water deprivation were delivered to both groups as part of the sucrose consumption test and that social isolation, another mild stressor, increases sucrose intake in mice, ${ }^{20)}$ we speculate that BI- $1^{-1-}$-control mice were more sensitive to the effects of food and water deprivation delivered before measuring sucrose consumption, at least transiently. Recently, Strekalova and Steinbusch $(2010)^{21)}$ suggested that sucrose preference is a parameter of the sucrose test, which makes absolute sucrose intake suitable for analyzing individual differences in hedonic sensitivity in mice. Despite this suggestion, we measured sucrose intake only because of inconsistent results for sucrose preference in our preliminary experiment (data not shown) due to the very small amount of water taken.

No significant difference in spontaneous locomotor activity was observed between BI- $1^{+/+}$-control and BI- $1^{+/+}$-stress groups, whereas the BI-1 ${ }^{-/-}$-stress group showed greater activity than the $\mathrm{BI}-1^{+/+}$-control group. These results suggest that CMS had no effect on locomotion in wild-type mice and that a BI-1 deficiency produced enhanced vulnerability to CMS. Anhedonia often accompanies psychomotor retardation in depressed patients. ${ }^{22)}$ Hence, we expected a decrease in locomotion in $\mathrm{BI}-1^{+/+}$-stress mice and a greater reduction in locomotion in $\mathrm{BI}-1^{-1-}$-stress 
mice, but we obtained the opposite findings. Inconsistent results have been reported regarding the effects of CMS on locomotion, including no effect, ${ }^{14,19,23)}$ a decrease, ${ }^{24-27)}$ and an increase. ${ }^{15,28,29)}$ These inconsistencies may be due to variations in light intensity, ${ }^{29)}$ temperature, or sound proofness of the experimental room. The exact meaning of hyperlocomotion in BI-1 $1^{-/-}$ -stress mice is unclear because increased locomotion can be interpreted as increased exploratory behavior toward a novel environment, a form of hyperactivity (anxiolytic-like behavior), or agitated behavior (anxiogenic-like behavior). This issue should be addressed in a future study using behavioral tests measuring anxiety such as an elevated plus maze, the dark-light exploration test, or the marble-burying test.

CMS increased immobility time during the FST in the $\mathrm{BI}-1^{+/+}$-stress group, which was similar to the results of many previous studies. It was interesting to see a greater increase in immobility time in the $\mathrm{BI}-1^{-/-}$-stress group than in the BI- $1^{+/+}$-stress group at week 2 , suggesting that BI-1 gene knockout may be associated with producing more severe depression-like behavior following CMS. This finding together with the greater reduction of sucrose consumption in the BI- $1^{-/-}$-stress group than in the BI- $1^{+/+}$-stress group at week 2 suggests that BI-1 has a protective function against the depressogenic effects of CMS in the short term, but not in the long term.

Several limitations of our study should be mentioned. First, it was the lack of baseline locomotor activity data. As interindividual variability in response to the stress of a novel environment often exists, this could be a confounding factor when comparing locomotor activities after CMS. Second, the sample size of each group was relatively small, and therefore there was a possibility of type II error. Additionally, our findings should be considered with related molecular studies to provide more insight into the role of BI-1 in depression. In conclusion, our results suggest the involvement of BI-1 in the production of depression-like behaviors following short-term CMS treatment.

\section{REFERENCES}

1. Bolduc N, Ouellet M, Pitre F, Brisson LF. Molecular characterization of two plant BI-1 homologues which suppress Bax-induced apoptosis in human 293 cells. Planta 2003;216:377-386.

2. Kawai-yamada M, Jin L, Yoshinaga K, Hirata A, Uchimiya H. Mammalian Bax-induced plant cell death can be downregulated by overexpression of Arabidopsis Bax Inhibitor-1 (AtBI-1). Proc Natl Acad Sci U S A 2001;98:12295-12300.

3. Xu Q, Reed JC. Bax inhibitor-1, a mammalian apoptosis suppressor identified by functional screening in yeast. Mol
Cell 1998;1:337-346.

4. Dohm CP, Siedenberg S, Liman J, Esposito A, Wouters FS, Reed JC, et al. Bax inhibitor-1 protects neurons from oxygen-glucose deprivation. J Mol Neurosci 2006;29:1-8.

5. Chae HJ, Kim HR, Xu C, Bailly-Maitre B, Krajewska M, Krajewski S, et al. BI-1 regulates an apoptosis pathway linked to endoplasmic reticulum stress. Mol Cell 2004;15: 355-366.

6. Bailly-Maitre B, Fondevila C, Kaldas F, Droin N, Luciano $\mathrm{F}$, Ricci JE, et al. Cytoprotective gene bi-1 is required for intrinsic protection from endoplasmic reticulum stress and ischemia-reperfusion injury. Proc Natl Acad Sci U S A 2006;103:2809-2814.

7. Bailly-Maitre B, Bard-Chapeau E, Luciano F, Droin N, Bruey JM, Faustin B, et al. Mice lacking bi-1 gene show accelerated liver regeneration. Cancer Res 2007;67:14421450 .

8. Kim HR, Lee GH, Ha KC, Ahn T, Moon JY, Lee BJ, et al. Bax Inhibitor-1 Is a pH-dependent regulator of $\mathrm{Ca} 2+$ channel activity in the endoplasmic reticulum. J Biol Chem 2008;283:15946-15955.

9. Baek D, Nam J, Koo YD, Kim DH, Lee J, Jeong JC, et al. Bax-induced cell death of Arabidopsis is meditated through reactive oxygen-dependent and -independent processes. Plant Mol Biol 2004;56:15-27.

10. Bachis A, Cruz MI, Nosheny RL, Mocchetti I. Chronic unpredictable stress promotes neuronal apoptosis in the cerebral cortex. Neurosci Lett 2008;442:104-108.

11. Silberman DM, Wald M, Genaro AM. Effects of chronic mild stress on lymphocyte proliferative response. Participation of serum thyroid hormones and corticosterone. Int Immunopharmacol 2002;2:487-497.

12. Ducottet C, Griebel G, Belzung C. Effects of the selective nonpeptide corticotropin-releasing factor receptor 1 antagonist antalarmin in the chronic mild stress model of depression in mice. Prog Neuropsychopharmacol Biol Psychiatry 2003;27:625-631.

13. Yalcin I, Aksu F, Belzung C. Effects of desipramine and tramadol in a chronic mild stress model in mice are altered by yohimbine but not by pindolol. Eur J Pharmacol 2005;514:165-174.

14. Elizalde N, Gil-Bea FJ, Ramírez MJ, Aisa B, Lasheras B, Del Rio J, et al. Long-lasting behavioral effects and recognition memory deficit induced by chronic mild stress in mice: effect of antidepressant treatment. Psychopharmacology (Berl) 2008;199:1-14.

15. Li S, Wang C, Wang M, Li W, Matsumoto K, Tang Y. Antidepressant like effects of piperine in chronic mild stress treated mice and its possible mechanisms. Life Sci 2007; 80:1373-1381.

16. Pothion S, Bizot JC, Trovero F, Belzung C. Strain differences in sucrose preference and in the consequences of unpredictable chronic mild stress. Behav Brain Res 2004; 155:135-146.

17. Matthews K, Forbes N, Reid IC. Sucrose consumption as an hedonic measure following chronic unpredictable mild stress. Physiol Behav 1995;57:241-248.

18. Forbes NF, Stewart CA, Matthews K, Reid IC. Chronic mild stress and sucrose consumption: validity as a model of depression. Physiol Behav 1996;60:1481-1484.

19. Ibarguen-vargas Y, Surget A, Touma C, Palme R, Belzung C. Multifaceted strain-specific effects in a mouse model of depression and of antidepressant reversal. Psychoneuroendocrinology 2008;33:1357-1368.

20. Coudereau JP, Stain F, Drion N, Sandouk P, Monier C, 
Debray M, et al. Effect of social isolation on the metabolism of morphine and its passage through the blood-brain barrier and on consumption of sucrose solutions. Psychopharmacology (Berl) 1999;144:198-204.

21. Strekalova T, Steinbusch HW. Measuring behavior in mice with chronic stress depression paradigm. Prog Neuropsychopharmacol Biol Psychiatry 2010;34:348-361.

22. Lemke MR, Puhl P, Koethe N, Winkler T. Psychomotor retardation and anhedonia in depression. Acta Psychiatr Scand 1999;99:252-256.

23. Li S, Wang C, Wang W, Dong H, Hou P, Tang Y. Chronic mild stress impairs cognition in mice: from brain homeostasis to behavior. Life Sci 2008;82:934-942.

24. D'Aquila P, Monleon S, Borsini F, Brain P, Willner P. Anti-anhedonic actions of the novel serotonergic agent flibanserin, a potential rapidly-acting antidepressant. Eur $J$ Pharmacol 1997;340:121-132.

25. Dang $\mathrm{H}$, Chen Y, Liu X, Wang Q, Wang L, Jia W, et al. Antidepressant effects of ginseng total saponins in the forced swimming test and chronic mild stress models of depression. Prog Neuropsychopharmacol Biol Psychiatry 2009;33:14171424.

26. Grippo AJ, Beltz TG, Johnson AK. Behavioral and cardiovascular changes in the chronic mild stress model of depression. Physiol Behav 2003;78:703-710.

27. D'aquila PS, Peana AT, Carboni V, Serra G. Exploratory behaviour and grooming after repeated restraint and chronic mild stress: effect of desipramine. Eur J Pharmacol 2000; 399:43-47.

28. Harris RB, Zhou J, Youngblood BD, Smagin GN, Ryan DH. Failure to change exploration or saccharin preference in rats exposed to chronic mild stress. Physiol Behav 1997;63: 91-100.

29. Strekalova T, Spanagel R, Dolgov O, Bartsch D. Stressinduced hyperlocomotion as a confounding factor in anxiety and depression models in mice. Behav Pharmacol 2005;16: 171-180. 